\title{
Réinventer l'harmonie politique de tous les êtres vivants : un projet révolutionnaire et zootechnique (1792-1820)
}

Re-inventing the Political Harmony of All Living Beings: A Revolutionary and Zoo-technical Project, 1792-1820

Die politische Harmonie aller Lebewesen neuerfinden. Ein revolutionäres Projekt aus dem Bereich der Tierzucht, 1792-1820

\section{Malik Mellah et Pierre Serna}

\section{(2) OpenEdition}

\section{Journals}

Édition électronique

URL : http://journals.openedition.org/rh19/5181

DOI : $10.4000 /$ rh 19.5181

ISSN : 1777-5329

Éditeur

La Société de 1848

Édition imprimée

Date de publication : 1 août 2017

Pagination : 31-46

ISSN : 1265-1354

Référence électronique

Malik Mellah et Pierre Serna, «Réinventer l'harmonie politique de tous les êtres vivants : un projet révolutionnaire et zootechnique (1792-1820) », Revue d'histoire du XIXe siècle [En ligne], 54 | 2017, mis en ligne le 01 juillet 2019, consulté le 02 janvier 2020. URL : http://journals.openedition.org/rh19/5181 ; DOI : 10.4000/rh19.5181 


\section{MALIK MELLAH PIERRE SERNA}

\section{Réinventer l'harmonie politique de tous les êtres vivants : un projet révolutionnaire et zootechnique (1792-1820)}

S'appuyant notamment sur les travaux de Keith Thomas ${ }^{1}$, Jocelyne Porcher relève l'apparition à partir de la fin du XVIII e siècle d'un conflit majeur entre les représentations des animaux et le rapport concret à la nature : «l'exploitation des animaux, conçue en tant que système est corollaire du développement d'une sensibilité nouvelle à l'égard des bêtes", écrit-elle ${ }^{2}$. Cette opposition culminerait dans les formulations contemporaines de la zootechnie, conçue comme science de l'exploitation des machines animales, et de la cause animale défendue lors de la fondation de la Société protectrice des animaux en 1845, puis du vote de la loi Grammont en 1850. Le bien-être humain serait construit aux dépens de celui des bêtes, non sans regret et dépit des âmes les plus généreuses. L'entrée dans l'âge industriel aurait à ce point modifié les relations entre les hommes et les animaux que la constitution de l'Homme s'appuierait alors davantage sur la destitution - physique et morale - de l'animal. La technique s'est emparée du vivant. Ce qui prime est la réalisation de performances au prix d'un double déni, de l'existence des animaux d'abord, de la sensibilité de ceux qui sont conduits à travailler avec ces bêtes maltraitées ensuite. Ce déni passe par le langage : il révèle l'assimilation du vocabulaire industriel conquérant par l'agriculture et un mimétisme assumé entre l'agronome et l'ingénieur. La machine triomphe de l'animal non seulement en le remplaçant progressivement mais encore par le nom et la norme. Ils ont également repoussé dans une pénombre la question du seuil de souffrance acceptable pour les animaux, puis la souffrance animale elle-même.

Réalité et discours se fondent largement sur les textes des zootechniciens qui, reprenant des normes des sciences et de l'industrie, décrivent désormais les bœufs, vaches ou chevaux comme «des machines au même titre que les locomotives de nos chemins de fer, les appareils de nos usines où l'on distille,

1. Keith Thomas, Man and the natural world: changing attitudes in England, 1500-1800, London, Allen Lane, 1983, traduction française Dans le jardin de la nature. La mutation des sensibilités en Angleterre à l'époque moderne (1500-1800), Paris, Gallimard/NRF, 1985. p. 177.

2. Jocelyne Porcher, Cochons d'or. L'industrie porcine en question, Versailles, Éditions Quae, 2010, 
où l'on fabrique du sucre, de la fécule, où l'on tisse, où l'on moud, où l'on transforme une matière quelconque. Ce sont des machines donnant des services et des produits ${ }^{3}$. Des discours se construisent et se diffusent largement, jusqu'à peupler les imaginaires et les fonds d'archives : ce sont en grande partie eux ou les réactions qu'ils suscitent qui nourrissent la construction historiographique du Point de vue animal, de cette «autre version de l'histoire» qui intègre dans le récit historique les apports les plus féconds de l'éthologie ou de l'anthropologie pour relire du côté des animaux une histoire... avant tout humaine et politique. Au nom de la subjectivité et de l'agency animale, cette histoire aurait pourtant encore à voir avec une vue mécanique de l'animal ${ }^{4}$.

Dans ce cadre, la période de la première République (1792-1804), a des difficultés à trouver sa place dans la littérature née souvent de la cause animale, sinon à nourrir l'hypothèse d'un décalage grandissant entre les sensibilités et la réalité de l'exploitation, ce que Jean-Yves Bory affirme lorsqu'il ancre le "paradigme vivisecteur» dans "une autre religion [qui] a émergé à la fin du XVIII ${ }^{e}$ siècle et [qui] paraît avoir eu une influence plus déterminante [que la religion catholique], c'est le messianisme scientifique mis en œuvre par la Révolution de 1789, véritable "religion nouvelle» selon François Furet ${ }^{5}$. Il existe un point aveugle dans cette littérature : au mieux la décennie révolutionnaire se fige en un "échec prometteur", en une annonce des initiatives et des idées ultérieures, paradoxaux puisque ne faisant que reprendre des débats toujours anciens autour de la brutalité ou de la violence ${ }^{6}$.

La constitution d'une zootechnie "productiviste» relève de choix. Des choix qui repoussent dans une pénombre épaisse les alternatives tant sont grandes la propension ou la capacité de l'élevage productiviste à dire ce qu'ont été l'histoire et le progrès "agricoles", tant est puissante également l'inclination à investir l'histoire de la zootechnie à partir du rejet des productions animales ou d'une nouvelle mort de masse ${ }^{7}$. Revenir ici sur l'animal en Révolution ne se limite pas à faire de l'animal un «Autre» qui rejoindrait l'esclave, le colonisé ou la femme dans la liste de ceux qui ont extirpé une voix et une histoire en même temps que leur liberté et leur égale dignité. Il s'agit encore moins de revenir sur la part animale d'un peuple zoomorphe. La Révolution n'a jamais pensé l'égalité de l'homme et de l'animal mais s'est constamment inquiétée de l'égalité de l'homme et de ses semblables. Il s'agira donc ici d'explorer un autre "chemin qui n'a pas été pris» dans une époque qui a d'abord redessiné l'espace des possibles et qui finit en se figeant

3. André Sanson, Traité de zootechnie, Librairie agricole de la Maison rustique, tome 1, 1907 (5 édition revue et corrigée), p. 4.

4. Éric Baratay, Le point de vue animal. Une autre version de l'histoire, Paris, Seuil, 2012.

5. Jean-Yves Bory, La douleur des bêtes, la polémique sur la vivisection au XIX'e siècle en France, Rennes, PUR, 2013, p. 109.

6. Éric Baratay, "La promotion de l'animal sensible : une révolution dans la Révolution ", Revue historique, 1/2012, $n^{\circ}$ 661, p. 131-153.

7. Cf., parmi bien d'autres ouvrages de Charles Patterson, Un éternel Treblinka, Pairs, CalmannLévy, 2002. 
autour de sciences fixistes, de l'inégalité absolue du retour à l'esclavage et de la légitimation de la domination sur des «biens meubles" qu'on devine sensibles dans les appartements bourgeois et dont on ignore les souffrances dans les villes et les mines. Non sans avoir laissé les traces, éparses, d'une pensée républicaine, l'évolution des relations entre homme et animal illustre autant qu'elle explique les mutations économiques et techniques les plus profondes. L'animal est un façonneur de Cité dans toutes les dimensions de cette expression $^{8}$. Il reste néanmoins un acteur animal.

Cet article se propose de présenter une synthèse en marche, à partir de travaux déjà publiés et de discussions en cours. Il affirme que la période qui va des années 1780 aux années 1820 fut porteuse d'une autre proposition zootechnique née du noud de fondations et de contradictions politiques? Autour des écrits de Jean-Baptiste Salaville, nous reviendrons d'abord sur l'existence d'une pensée républicaine du rapport à l'animal qui ne serait le produit ni d'une volonté conservatrice, ni d'un simple réagencement des réflexions laïques ou religieuses sur la violence faîtes aux animaux. Il sera ensuite question d'une relecture de programmes et de réalisations qui ont en commun une volonté de réenchanter le monde et de républicaniser les rapports de l'homme au monde naturel : dans les ménageries, dans la définition d'une économie politique républicaine et dans le projet des Idéologues.

\section{UNE PENSÉE RÉPUBLICAINE DE L'ANIMAL}

\section{Le concours de l'Institut en 1802 ou comment éradiquer la barbarie à visage humain}

Une pensée «républicaine» de l'animal s'élabore dès la naissance du régime en 1792. Cette conception prend en considération l'animal dans le cercle d'une citoyenneté qui s'est élargie jusqu'à l'impensable, l'inimaginable : élever les esclaves noirs au rang de citoyens de la République. La question se pose donc naturellement en un double sens aux finalités radicalement différentes, voire opposées. En élevant ceux qui n'étaient considérés que comme des sous-citoyens, dans une indétermination de bêtes de somme, la République peut-elle élever tous les vivants et reconsidérer le statut de tous les inférieurs sous les esclaves humains et donc des animaux? Ou bien, ques-

8. Pierre Serna, L'animal en République, 1789-1802. Genèse du droit des bêtes, Toulouse, Anarcharsis, 2016; Gregory Quenet, Versailles, une histoire naturelle, Paris, la Découverte, 2015; Clay Mc Shane et Joel A. Tarr, The Horse in the City, living in the Nineteenth Century, Baltimore, The Johns Hopkins University Press, 2007.

9. Les auteurs tiennent à remercier Jean-Luc Chappey, Sauvagerie et Civilisation. Une histoire politique de Victor de l'Aveyron, Paris, Fayard, 2017; Julien Vincent, "Par-delà nature et culture. L'animal des Idéologues, 1794-1804 ", à paraitre en 2017 dans les Annales Historiques de la Révolution Française, ainsi que Laurent Brassart, "La ferme des animaux ou l'invention d'une politique de l'animal utile sous le Consulat ", $A H R F, \mathrm{n}^{\circ} 377$, juillet-septembre 2014, p. 175-196; Boris Cattan et Bernard Gainot, pour les débats en cours au sein de l'Institut d'histoire de la Révolution française. 
tion dérangeante, en abaissant le seuil de reconnaissance de la citoyenneté, la République n'a-t-elle pas inventé les conditions de pensée d'un continuisme qui, en reconnaissant à l'animal une existence d'être vivant vient esquisser une zone obscure de confusion racialiste et dangereuse qui fera écrire huit ans après l'abolition de l'esclavage à ce même Virey, fort sensible au bien-être animal, que "le noir est au blanc ce que le singe est à l'homme ${ }^{10}$ ? Illustrant la première option, la pensée du républicain Salaville éclaire de façon pertinente la question des rapports politiques entre homme et non humain, entre citoyen et animal.

Le 17 messidor an X (6 juillet 1802), treize ans après la proclamation des Droits de l'Homme, le sujet mis au concours par la deuxième section de la classe des Sciences morales et politiques de l'Institut national propose de répondre à la question suivante pour son prix de morale : "Jusqu'à quel point les traitements barbares intéressent-ils la morale publique? Et conviendrait-il de faire des lois à cet égard?». La lecture des 27 dissertations envoyées à Paris montre une sensibilité nouvelle et une phobie de la violence qui expriment un héritage paradoxal de la Révolution pour cette génération de Français entrant dans le XIX ${ }^{e}$ siècle. Déboussolée par la guerre civile et le renversement de tous les principes anciens, la France doit se reconstruire à partir du ras du sol, à partir de l'organisation des plus insignifiantes créatures afin d'assigner à chacun sa place dans la République conservatrice de l'ordre restauré par le premier consul. Un mémoire tranche résolument avec tous les autres par la précision de son point de vue résolument républicain et démocrate à la fois, l'amenant à assumer pleinement, aux antipodes de la sensibilité des autres candidats, une position résolument "philanthrope», là où les autres participants au concours, opportunistes ou sincères, sont ouvertement zoophiles. Les animaux sont importants certes, mais les hommes, tous les hommes, de toutes les couleurs et de toutes les conditions, le sont davantage en 1802, année de rétablissement de l'esclavage. Assez de fausse sensiblerie, dit Salaville, puisqu'il s'agit de cet auteur, connu dans la République des lettres pour avoir été un des secrétaires de Mirabeau et un passeur de textes républicains radicaux du XVII siècle anglais dans l'édition française. La nature même du pacte social républicain, de par l'exigence intellectuelle qu'elle requiert de toutes les forces humaines, ne peut intégrer à un quelconque niveau l'animal... sans que cette position tranchée n'implique un tant soit peu une excuse pour les traitements barbares ${ }^{11}$.

Cependant, c'est ailleurs que Salaville avance une idée authentiquement originale, politique et subversive. Prenant les thèses qu'il ne partage pas, il les développe en toute logique et livre une intuition importante dont le lecteur

10. «Ils grimpent, sautent sur la corde, voltigent avec une facilité merveilleuse et qui n’est égalée que par les singes, leurs compatriotes, et peut-être leurs anciens frères selon l'ordre de la nature»: Julien Joseph Virey, Histoire naturelle du genre humain, Paris, Crochard, 1824.

11. Pierre Serna, L'animal en république, op. cit., p. 205-220. 
saisit qu' elle va concerner le statut des noirs redevenus esclaves, par un biais différent des autres dissertations qui ont mentionné le problème. Ainsi, «si les animaux sont ce que nous sommes, il n'y a d'eux à nous d'autre différence que du plus au moins, s'il est encore vrai que cette différence nous autorise, à les traiter comme nous les traitons, pourquoi n'en serait-il pas de même dans notre espèce? N'y a-t-il pas du plus et du moins parmi les hommes? „ ${ }^{12}$. L'acceptation d'un lien de nature entre les animaux et les hommes est le premier degré qui permet de penser, dans ce contexte particulier, une lente gradation de l'ordre des primates à celui des hommes. Cette idée conduit également à accepter l'idée d'une inégalité constitutive entre les hommes, et à terme, à justifier l'injustifiable, pour le Républicain Salaville, la mise en servitude de certains humains avec le rétablissement de l'esclavage depuis 1802, ou la construction de l'inégalité entre les hommes, fondement du racialisme de la première moitié du XIX siècle $^{13}$.

Ce n'est pas un des moindres mérites de ce mémoire republié sous forme de livre en 1805, sous le titre explicite De l'homme et des animaux, que d'avoir su proposer une position politique audacieuse. De la différence soutenue entre humains et bêtes, vrai débat d'un point de vue scientifique, nait un point de vue politique suggérant une remise en cause de l'organisation sociale fondée sur la certitude de la faiblesse quasi naturelle de groupes humains défavorisés, et sur la naturelle mise en servitude des peuples africains ${ }^{14}$.

\section{La ménagerie républicaine, un spectacle citoyen}

Salaville ne peut que regretter les dix années entre 1792 et 1802 , où l'invention de la République a été suivie d'une série de projets et de réalisations visant à réenchanter le monde malgré la guerre et la violence du temps, à républicaniser les rapports de l'homme au monde vivant, à commencer par les animaux ${ }^{15}$. Ainsi sur proposition de Lakanal, La Convention vote, le 10 juin 1793, le décret de fondation d'un Museum d'histoire naturelle intégrant officiellement la zoologie aux savoirs des naturalistes ${ }^{16}$. Le 27 floréal an II (16 mai 1794) le Comité de salut public ordonne «de faire arranger provisoirement quelques loges pour les animaux dans le lieu et le terrain le plus convenable... et se servant des grilles de fer et des cages appartenant

12. Salaville, De l'homme..., op. cit., p. 16-17.

13. Claude-Olivier Doron, L'homme altéré, race et dégénérescence, XVIII'-XIXe siècle, Ceyzérieux, Champ Vallon, 2016.

14. Claude Benichou et Claude Blanckaert, Julien-Joseph Virey, naturaliste et anthropologue, Sciences en situations, Paris, 1992; Claude Blanckaert, "Fondements disciplinaires de l'anthropologie française au XIX e siècle. Perspectives historiographiques", Politix, 1995, n 29, p. 31-54.

15. Yves Laissus et Jean-Jacques Petter, Les Animaux du Museum. 1793-1993, Paris, Imprimerie nationale, et Emma Spary, «Le spectacle de la nature : Contrôle du public et vision républicaine dans le Museum jacobin ", in Claude Blanckaert, Claudine Cohen, Pietro Corsi, Jean-Louis Fischer [dir], Le Muséum au premier siècle de son histoire, Éditions du Muséum d'histoire naturelle, 1997, p. 457479.

16. Jean-Luc Chappey. Des naturalistes en Révolution. Les procès-verbaux de la société d'histoire naturelle de Paris (1790-1798), Paris, CTHS, 2009. 
à la ci-devant ménagerie de Versailles ${ }^{17}$. Entre-temps les commissaires de Paris, sur décision des administrateurs du département, prise le 14 brumaire an II (4 novembre 1793), ont reçu l'ordre de débarrasser la capitale des spectacles d'animaux, dégradants pour les citoyens et les bêtes qui doivent être amenées et déposées contre indemnisation au Jardin des Plantes ${ }^{18}$. Pourtant, 65 mammifères et 25 oiseaux meurent à la fin de 1794 faute de soin et de nourriture. Antoine Clair Thibaudeau, député Thermidorien, qui figure parmi les principaux artisans de la Constitution de l'an III, ne croit pas déroger lorsqu'il s'en préoccupe au nom des Comités d'Instruction publique et des Finances devant la Convention le 21 frimaire an III (11 décembre 1794). Il s'agit de convaincre les députés de voter des subsides pour réserver un meilleur sort aux animaux. Thibaudeau retrace l'histoire du jardin des plantes médicinales, puis du cabinet d'histoire naturelle fondé par Buffon avec l'aide de Daubenton. Il explique à ses collègues qu'il ne faut pas faire de la ménagerie une prison pour animaux, il ne faut pas affliger le public du spectacle de l'esclavage et de la flétrissure de la nature. Il faut, au contraire, instruire le public des citoyens et garantir l'ordre public en offrant un spectacle édifiant pour les vertus et la morale républicaine.

Le rapport entre le public et les animaux dans le Nouveau Régime républicain, nourrit nombre d'autres textes, en particulier autour de la nouvelle ménagerie. Celle-ci est installée dans ce quartier pauvre de Paris, comme un choix politique assumé d'édifier un lieu de sciences dans une zone délaissée de la périphérie urbaine, proche du marché aux bois et de la remise des coches de la capitale. Parmi ces auteurs d'un genre nouveau, politique et scientifique à la fois, Bernard de Lacépède jouit dans les nouvelles institutions d'un réel prestige ${ }^{19}$. Depuis 1795 et la refondation de la République après la Terreur, il a, avec d'autres savants, construit le projet de la ménagerie autour de principes forts : placer les animaux dans un espace propre et salubre, les acclimater à leur nouvelle vie, avantager leur propagation, les faire multiplier, améliorer leur race et refonder une hiérarchie du vivant et du sensible ${ }^{20}$. N'est-ce pas le programme de la république fondée sur les bonnes mœurs, sur l'organisation d'un ordre public assignant à chacun sa place, valorisant les citoyens-travailleurs, canalisant les citoyens brutaux, éduquant les citoyens enfants, dans une urbanisation nouvelle ${ }^{21}$ ? Ainsi pensées, les ménageries sont essentielles par leur objet et parce qu'elles constituent de

17. Archives nationales (Arch. nat.), Aj 15844 : «État des animaux vivans, existants à la ménagerie provisoire du Museum national d'histoire naturelle», 26 Floréal an II (15 mai 1794).

18. Archives de la préfecture de Police de Paris / Section des Arcis AA 59, 17 brumaire an II, "Saisie de bêtes fauves sur la place de la maison commune».

19. Claude Blanckaert, "Une anthropologie de transition : Lacépède et l'histoire naturelle de l'homme (1795-1830)", Annales Benjamin Constant, $\mathrm{n}^{\circ}$ 13, 1992, p. 95-211.

20. Lacepède, Euvres complètes, 1826, Discours, p. 345.

21 Gregory Quenet, Qu'est-ce que l'histoire environnementale?, Seyssel, Champ Vallon, 2014. 
parfaits miroirs de l'état de progrès auquel sont parvenues les sociétés qui ont eu l'idée de constituer ces "rassemblements d'animaux $»^{22}$.

L'histoire des ménageries se confond en effet pour Lacépède avec l'histoire du monde. Son texte réédité plusieurs fois dans les nombreux guides publiés sous le consulat et l'Empire, la visite constante et toujours plus populaire de la ménagerie jusqu'au triomphe de la girafe arrivée au printemps 1827, et visitée par des milliers de Parisiens, ancrent le parc animalier dans l'imaginaire mental des Parisiens et des touristes ${ }^{23}$. La France, nation libre, doit emprunter le chemin de la propédeutique grecque et s'inspirer de l'exemple d'Alexandre qui envoyait les animaux à Aristote pour qu'il les étudie. Cette vision est affinée dans la préface de La Ménagerie du Museum, ouvrage écrit avec Cuvier et Geoffroy et illustré avec maestria par Maréchal, le peintre animalier. Lacepède y précise une vision de l'histoire divisée en cinq périodes. La cinquième, contemporaine de la ménagerie républicaine, couronne l'ensemble lorsqu'une pensée de la régénération, commune à celle des hommes, devient le programme politique des responsables du «bienêtre» des animaux. Dans une perspective proche de l'économie rurale, la ménagerie doit veiller à l'amélioration des races d'animaux : en domestiquant les bêtes féroces, en acclimatant les animaux étrangers, en améliorant les troupeaux d'animaux travailleurs ou en observant ceux attachés par l'affection à l'homme. La ménagerie devient un laboratoire de la construction de la relation citoyen / animal. Lacepède saisit bien le sens "philosophique, patriotique et scientifique» du face-à-face entre les bêtes et les hommes, dans une nouvelle République.

La ménagerie offre cet avantage unique d'être une république dans la cité et de renvoyer confusément l'ordre parfait d'un régime encore naissant. Ce n'est pas tout de conserver les animaux et de les étudier dans une captivité douce, le but ultime consiste en la vérification matérielle que le projet d'une ménagerie républicaine fonctionne parce qu'elle est pensée et édifiée selon une finalité différente : la reproduction des animaux. La naissance des animaux dans le monde fermé qu'est la ménagerie doit servir de preuve de la réussite du modèle scientifique républicain. Ainsi, les naturalistes de la ménagerie ne cachent-ils pas leur fierté lors de la naissance de trois lionceaux en $1800^{24}$. Ils les dénomment Fleurus, Jemmapes, et Marengo, esquissant toute une symbolique qui ne saurait échapper au public qui se rend en masse les observer $^{25}$. Le citoyen soldat affirme sa virilité par sa fécondité, tout comme

22. Article de 1795/ réécrit en 1801, désormais référencé dans l'édition de 1826, p. 340.

23. La ménagerie du muséum d'histoire naturelle ou description et histoire des animaux qui y vivent et qui y ont vécu. Par Lacépède, Cuvier et Geoffroy, avec des figures peintes par Maréchal peintre du muséum, à Paris, chez Miger et Renouard, an XII, 1804. Voir l'article d'Éric Baratay dans ce numéro.

24. Peu auparavant, l'échec du couple d'éléphants, dont la tentative d'accouplement avait pourtant été organisée avec le plus grand soin par les naturalistes, avait passionné les savants et les lecteurs de la presse parisienne : J. P. Houel, Histoire naturelle des deux éléphants, mâle et femelle, du Muséum de Paris, venus de Hollande en France en l'an VI, Paris, Huzard, an XII, 1803.

25. E. J. Vignier, Phénomène d'histoire naturelle. Récit de la deuxième gestation de Constantine une 
le couple de lions acheté à Londres et stérile au pays de sa majesté, est devenu «républicainement» fécond dans la ménagerie de Paris ${ }^{26}$. La République a inventé un citoyen et une citoyenne nouvelle, sains, féconds. Les lions ne sont plus des rois mais des citoyens rebaptisés civiquement et Lacepède de rappeler que la ménagerie est l'antichambre d'un monde bien ordonné qui passe par le souci de l'économie politique et son utilitarisme.

\section{UNE ÉCONOMIE POLITIQUE RÉPUBLICAINE}

L'invention du citoyen fécond a aussi à voir avec une économie politique agricole. "L'œil du maître engraisse le bétail», écrit sous la Restauration le savant Arsène Thiébaut de Berneaud qui veut renouer avec une pensée économique et politique de l'animal domestique héritée de la Révolution :

"On remarque effectivement une grande différence entre les animaux soignés, je dirai amicalement, et ceux qui sont abandonnés à la solitude ou maltraités. Les premiers semblent s'honorer de la part que l'homme leur accorde dans ses travaux; les seconds au contraire, sont avec lui dans un état de guerre perpétuelle : s'ils cèdent, c'est à la violence; mais dès qu'ils retrouvent leurs forces, leur énergie première, ils sont plus dangereux encore. La contrainte irrite l'animal le plus doux; l'aiguillon, le fouet, les coups d'éperons l'avilissent, le rendent rétif, mutin et méchant ${ }^{27}$.

La pensée dont il se réclame est celle de "l'économie rurale», une véritable proposition d'économie politique qui nourrit le projet républicain à partir de septembre 1792. La fondation de la République et la régénération des mœurs doivent correspondre à un retour des esprits vers l'agriculture et des attentions envers les animaux de proximité. Le citoyen-Cincinnatus de l'an II doit combattre les ennemis de la République, mais aussi l'inertie et les routines pour créer les conditions d'une économie vertueuse : celle d'une république agricole, moderne, prospère et apaisée ${ }^{28}$. «Faisons naître autour de nous les animaux utiles, et soignons nos troupeaux : en vivant près d'eux, nous y éprouverons bientôt des sentimens intéressans, nous les connaitrons

des lionnes de la ménagerie du Jardin des Plantes, précédé de la description de tous les quadrupèdes sous la garde du citoyen Cassal, Paris, Quilleau, an IX, p. 30.

26. On comprend pourquoi dans les instructions qui sont données aux garçons de la ménagerie, l'observation minutieuse des accouplements des gestations des accouchements, des allaitements des sevrages est recommandée avec la plus grande attention. $C f$., MMS «Observations particulières sur les animaux de la ménagerie, an XII-1822».

27. Arsène Thiébaut de Berneaud, Traité de l'éducation des animaux domestiques dans lequel on indique les moyens les plus simples et les plus surs de les multiplier, de les entretenir en santé, et d'en tirer le plus d'avantages possibles, tome premier, Paris, Audot, 1820, p. 5.

28. James Livesey, Making Democracy in the French Revolution, Cambridge (Mass.), Harvard University Press, 2001, p. 89-92. 
mieux; et que de choses leurs mœurs naïves et douces nous apprendrons! $\aleph^{29}$. Sous la plume du Conventionnel Coupé de l'Oise, comme dans les discours d'autres élus, savants ou agronomes, créer une science de la conservation et de l'amélioration des animaux domestiques devient un enjeu républicain, depuis les ménageries du Muséum d'histoire naturelle jusqu'aux troupeaux de la République. En 1795, Les Écoles vétérinaires d'Alfort et de Lyon sont réorganisées en écoles "d'économie rurale et vétérinaire», les domaines de Rambouillet ou du Raincy rassemblent des animaux destinés à "épurer» les troupeaux français et la classe des sciences physiques et mathématiques de l'Institut accueille des vétérinaires dans sa section d'économie rurale et d'art vétérinaire. La fondation de la République se manifeste également par la formulation d'une première zootechnie dont il convient de faire l'archéologie.

\section{L'économie rurale et les bergers}

Installé à partir de 1783 dans la première chaire d'économie rurale instituée en France, le naturaliste Louis-Jean-Marie Daubenton est alors l'auteur célébré de l'Histoire naturelle des animaux de L'Encyclopédie méthodique ${ }^{30}$. Il entend mettre en ouvre un programme de recherches qu'il introduit par un «Mémoire sur l'économie vétérinaire et rurale». Il y fixe les horizons du champ vétérinaire : œuvrer "pour le maintien de la société et j'ose dire pour le bonheur des hommes " ${ }^{31}$. Ce qui intéresse alors le savant, c'est d'étendre l'emprise de l'homme, sa capacité à intégrer l'animal au progrès de la société. Il est possible de rattacher ce que Daubenton entend développer à la zootechnie, définie par André Sanson comme «l'art d'exploiter économiquement suivant les principes de la science, de tirer des diverses espèces du genre le meilleur parti, tout à la fois pour celui qui les exploite et pour la société» ${ }^{32}$.

Étendre l'emprise humaine, c'est d'abord élargir «l'économie vétérinaire ${ }^{33}$. Tout animal peut être utile à l'homme de quelque classe qu'il soit, du quadrupède à l'insecte. Cette extension consiste alors dans un vaste programme d'acclimatement et d'observations intégré dans un discours historique, rappelant les travaux de Varron et de Columelle. Il le replace surtout dans une pensée scientifique qui affirme que "parmi les productions de la nature, l'homme ne peut modifier que les êtres organisés qui sont les animaux et les végétaux [...] Nous tirons des blocs de marbre du sein de la terre, nous leur donnons des formes et du poliment par notre travail, mais

29. De la détermination à donner à l'instruction publique vers l'agriculture par J.-M. Coupé (de l'Oise), imprimé par ordre de la Convention nationale, vendémiaire an III (septembre-octobre 1794).

30. Louis-Jean-Marie Daubenton, «Introduction à l'histoire naturelle», Encyclopédie méthodique, Paris, Panckoucke, 1782, volume I.

31. Arch. nat., F10 1194 mémoire de L.J.M. Daubenton. Toutes les citations du naturaliste qui suivent sont issues de ce mémoire.

32. André Sanson, Les moutons, histoire naturelle et zootechnie, Paris, Librairie agricole de la maison rustique 1868 , p. $78-79$.

33. Malik Mellah, "Portrait du berger en figure républicaine», Annales historiques de la Révolution française, $\mathrm{n}^{\circ} 374$, octobre-décembre 2013, p. 89-90. 
nous n'avons aucune influence sur leurs couleurs, ni sur le degré de leur dureté», contrairement aux êtres organisés qui reçoivent de nouvelles qualités à mesure que l'homme les «soigne».

Soutenu par un mouvement agronomique qui promeut l'économie rurale, Daubenton rassemble dans l'École vétérinaire d'Alfort une ménagerie "économique» et influence les premiers grands vétérinaires, Pierre Flandrin, Philibert Chabert et surtout François-Hilaire Gilbert. Cette pensée nourrit un projet qu'on ne peut limiter à sa dimension économique tant sont grandes les imbrications entre savoirs naturalistes, agronomie et pensée politique. C'est ce qu'exprime Gilbert au mois d'avril 1789, lorsqu'il revendique devant des autorités du royaume venues à Alfort pour assister au concours des élèves quelques quartiers de noblesse pour les animaux domestiques : «ils ont d'ailleurs assez d'autres caractères de noblesse qu'on ne peut leur contester ${ }^{34}$. Au-delà, l'économie vétérinaire se répand dans les cercles politiques et philosophiques. Le vénérable Daubenton, devenu instituteur républicain, la présente devant une assistance prestigieuse, au seuil de la Révolution à Alfort puis dans ses cours à l'École normale en l'an III ${ }^{35}$. On la retrouve chez l'abbé Grégoire dans son Essai historique sur l'état de l'agriculture en Europe au seizième siècle inséré dans la grande édition du Théâtre d'agriculture d'Olivier de Serres, initiative née de la conviction des élites du Directoire et poursuivie sous le Consulat. Pas plus le sujet que la distance historique ne doivent tromper sur le projet politique de Grégoire qui demeure fermement républicain. L'économie vétérinaire est d'abord une action physique et morale. «Malheur à quiconque voudrait placer ces observations hors du cercle des idées morales ", menace Grégoire après avoir disserté sur la négligence des propriétaires, sur l'horreur des combats d'animaux, sur la cruauté vis-à-vis des abeilles que l'on tue après avoir récolté le miel, ou vis-à-vis du rossignol qui paie de ses yeux les beaux trilles qu'on lui arrache ${ }^{36}$.

La sensibilité comme la douleur des animaux ne sont pas méconnues. Elles ne relèvent pas du fantasme ou de la littérature. On en trouve l'indication nette sous la plume de Daubenton dans l'Encyclopédie méthodique qui, grand anatomiste, perçoit et analyse la souffrance des bêtes par l'économie des organes et la sensibilité de l'animal : "les animaux, du moins ceux qui ont des sens, de la chair, et du sang, sont des êtres sensibles; comme nous ils sont capables de plaisir et sujets à la douleur [...] La pitié naturelle est fondée sur les rapports que nous avons avec l'objet qui souffre; elle est d'autant plus vive que la ressemblance, la conformité de naturel est plus grande; on souffre en

34. Arch. dép. Val-de-Marne (Archives départementales du Val-de-Marne), 1 ETP 2206, discours de François-Hilaire Gilbert.

35. Séances des écoles normales recueillies par des sténographes, nouvelle édition, Paris, à l'Imprimerie du Cercle social, 1800 (an IX de la République française), tome I, p. 106-112.

36. Grégoire, "Essai historique sur l'état de l'agriculture en Europe au seizième siècle», Introduction à l'édition du Théâtre d'agriculture et message des champs d'Olivier de Serres, nouvelle édition, à Paris, Huzard, an XII (1804), tome I, p. clvi. 
voyant souffrir son semblable $»^{37}$. Grégoire, pour sa part, rappelle l'évidence qu'il est plus facile d'épargner des douleurs inutiles aux animaux que l'on exploite et que l'on tue que «de faire adopter le système de Descartes qui les assimile aux automates».

Quel «Autre» est alors cet animal? Gilbert, parmi ces savants, se refuse à ranger l'homme "dans la même classe que les animaux, la primatie du premier sur le second ne sera jamais douteuse qu'à ceux qui, partisans avides de toutes les opinions singulières, croient que la grandeur de l'esprit humain consiste à paraître supérieur à la raison et à la vérité ${ }^{38}$. Pour ce savant, comme pour Grégoire, l'amélioration et l'exploitation des animaux entraînent des devoirs envers les paisibles compagnons des travaux humains : "quel est, Messieurs, l'homme assez ingrat ou assez borné pour ne pas reconnaitre les obligations qu'il a à ces derniers? ${ }^{39}$. Comment un propriétaire ne pourrait-il pas partager «le mouvement de sensibilité qu'inspire la vue de l'oie stupide, qui fait mouvoir la roue du tournebroche pour rôtir un individu de son espèce? $»^{40}$. Ces devoirs ne peuvent provenir de droits puisque si les animaux «donnent quelquefois à l'homme des secours salutaires et trop peu sentis, ce n'est peut-être que l'effet de la contrainte qui leur a été imposée par la nature, et je ne veux point leur faire un mérite des actes qu'ils ne produisent que par défaut de liberté». Ces "obligations» irrigueront une bonne partie de la littérature agronomique de la période, cette même littérature qui appelle à étendre l'emprise de l'homme.

\section{De l'art d'exploiter "républicainement" les animaux}

Dans cette «archéologie» de la zootechnie, un second courant se superpose à l'économie vétérinaire : c'est celui qui, né des pénuries d'animaux dans la crise de l'an II, vise à créer une science républicaine de l'animal vivant. Son projet est d'étendre la "bonté" pour transformer la société, c'est-à-dire d'exploiter par le soin, l'alimentation, la perfectibilité des animaux : «Il faut donc qu'un cultivateur éclairé se distingue par la bonté de ses chevaux, de ses bœufs, de ses vaches, de ses cochons, comme par la bonté de ses blés, de ses fruits, de ses légumes, etc. $»^{41}$ La bonté est selon le naturaliste Louis-Augustin Bosc le but vers lequel doit tendre le perfectionnement des animaux : elle " est presque toujours la compagne de la bonté, et la bonté morale s'unit souvent dans les animaux avec la bonté des services qu'on en attend ». Cet objet est repris largement dans l'édition de 1809 du Cours complet d'agriculture qui

37. Louis-Jean-Marie Daubenton, «Introduction à l'histoire naturelle», Encyclopédie méthodique, op. cit. p. 253-254.

38. Arch. dép. Val-de-Marne, 1 ETP 2206, discours de François-Hilaire Gilbert.

39. Ibidem.

40. Grégoire, «Essai historique sur l'état de l'agriculture en Europe au seizième siècle», op. cit., tome 1, p. clvi- clviii.

41. Bosc, "Bonté", Nouveau cours complet d'agriculture théorique et pratique, Paris, Deterville, 1809 , tome 2, p. 397. 
s'ouvre par un long texte de Parmentier dans lequel la condition de l'animal devient le marqueur du progrès de la République dans les campagnes.

C'est d'abord aux techniciens et aux savants que Parmentier s'adresse. C'est à eux qu'il appartient de "donner l'impulsion vers l'éducation des troupeaux et le perfectionnement des races : "rien n'est plus nécessaire au maintien de la santé et de la vigueur des animaux domestiques que les bons traitemens" ». "Assujettis», "expropriés", «éloignés de la nature», ils méritent bien, ces "amis de l'homme", qu'on paie de quelques soins les peines que les besoins humains leur imposent. Aux prisons que sont cabanes et étables, le savant préfère les «bienfaits de l'hospitalité" puisqu'ils en "sentent également les douceurs». Tout ceci, le savant le justifie par la nature de l'animal : «nous leur supposons de l'instinct, et même de l'intelligence, et nous nous refusons de croire qu'ils sont doués d'une sensibilité qui s'irrite contre la rigueur et qui sait apprécier la bonté! ${ }^{42}$.

L'art d'exploiter économiquement les animaux résulte de l'association de deux améliorations : développer les facultés physiques des bêtes par la diffusion des nouveaux principes sur les croisements ou l'hygiène vétérinaire, et s'appuyer sur le développement des facultés morales du bétail. Il faut profiter de l'instinct dont sont doués les animaux pour créer des habitudes heureuses et rompre les "inclinaisons dépravées $»^{43}$. On doit les accoutumer insensiblement aux travaux auxquels ils sont destinés. Il convient d'écarter les hommes brutaux ou insouciants qui martyrisent leurs compagnons animaux. Il faut ensuite considérer la nature des animaux, accepter par exemple le pas lent du bœuf ${ }^{44}$. Surtout, Bosc appelle à profiter de leurs capacités, y compris morales telle celle de s'attacher aux personnes qui les soignent. S'agit-il de préconisations hasardeuses? «Voyez la Suisse, l'Angleterre et autres cantons où on traite les chevaux, les bœufs, les vaches avec douceur, et où on en tire un service plus considérable qu'ailleurs! ${ }^{45}$. La science de l'animal vivant doit encore résulter de l'entreprise pédagogique et «thérapeutique» que constitue la dynamique de régénération républicaine. La culture des "élites" qui promeuvent l'action sur l'animal, qui cherchent à agir pour transformer les conditions d'existence des populations, ne se construit pas systématiquement dans une opposition réductrice à un peuple qu'il faudrait civiliser. Une partie du savoir diffusé par François-Hilaire Gilbert ne provient-il pas des questionnaires et des enquêtes qu'ils mobilisent au plus près de ceux qui sont en charge des animaux ${ }^{46}$ ?

La renommée des savants peut également tenir à leur considération pour le sort de celui qui travaille et qui souffre, c'est-à-dire aussi l'infortuné com-

42. Parmentier, Discours préliminaire, ibidem, tome I, p. xxvi.

43. Parmentier, «article hygiène", ibidem, op. cit. p. 182.

44. Bosc, «aiguillon", ibidem, tome 1, p. 176.

45. Bosc, "attachement», ibidem, tome 2, p. 97.

46. Arch. dép. Val-de-Marne, 1 ETP 2208 : «Projet d'expérience pour parvenir à la connaissance de la nature de la marche des causes et du traitement de l'épizootie régnante». 
pagnon animal. Ainsi lit-on de Parmentier que «sa sensibilité s'étendait jusque sur les animaux. Il recommandait aux cultivateurs de bien traiter les paisibles compagnons de leurs travaux, de ne plus souffrir qu'on les entassât dans de chétives étables, dans des écuries basses et obscures [...] et d'être bien persuadés que ceux qui exercent la cruauté sur les brutes n'ont point d'humanité» ${ }^{47}$.

Ces idées se diffusent. Loin de Paris, L. Chambert, un vétérinaire peut en nourrir un Essai sur l'amélioration des principaux animaux domestiques ${ }^{48}$, ouvrage technique et pratique qui constitue aussi un véritable appel à davantage d'humanité vis-à-vis des animaux : «Des animaux si précieux, les voir ainsi oubliés et maltraités! [...] Traitez-les avec ménagement : ils l'exigent de vous, ils seront reconnaissants de vos soins ». Il en appelle au ménagement, à la précaution. Et malheur aux hommes durs, impitoyables et barbares : «un juste châtiment vous attend; vous gémirez sur votre insouciance et sur vos emportements passés en regrettant trop tard de vous y être livrés» : d'abord la ruine économique, enfin des tourments ${ }^{49}$. L'auteur est distingué par diverses sociétés savantes : son discours n'est pas marginal. Il tend à former un idéal civil impliquant que le progrès économique et le bien-être des animaux peuvent n'être en concurrence ni dans les faits ni dans les esprits. Pourtant, l'historien ne peut méconnaître que les conditions mêmes d'organisation des guerres incessantes entre 1792 et 1815 , avec les levées multiples de chevaux, sans même évoquer leur utilisation industrielle, dévoreuse de bien d'autres animaux, ont contribué à une exploitation accrue des bêtes... de somme ${ }^{50}$.

\section{Le moment idéologique prolonge et actualise la zootechnie républicaine}

Dans un ouvrage débuté en l'an III et resté inédit jusqu'en l'an XII, Pierre-Jean Georges Cabanis inscrit les animaux domestiques dans une communauté du travail et du soin dont l'objectif est de «renouer la trame du bonheur ". La médecine est mise au service de la société évidemment par son influence immédiate mais surtout par ses lumières et par les perfectionnements physiques et moraux qu'elle offre. Comme les plantes qu'on voit acquérir de nouvelles qualités sous la main de l'habile cultivateur, «l'animal $[\ldots]$ nourri, cultivé, dressé, systématiquement par l'homme, acquiert des dispositions nouvelles et entre dans une nouvelle série d'habitudes» qui

47. Philippe Mutel, Vie d'Antoine-Augustin Parmentier, Paris, chez madame Huzard, 1819, p. 59-60; Éloge de Parmentier par L.-F. Grognier, Paris, Huzard, 1823; Antoine Miquel, Éloge de Parmentier, discours qui a remporté le double prix proposé par l'Académie d'Amiens, pour l'année 1819, Paris, Bureau de la Gazette de santé, Imprimerie de Hocquet, 1823.

48. L. Chambert, Essai sur l'amélioration des principaux animaux domestiques dans le département de la Charente-Inférieure; applicable, par ses principes, à beaucoup d'autres lieux: ouvrage utile aux agriculteurs, aux hommes de cheval, aux commerçants et engraisseurs du bétail, tome premier, Paris, Huzard, 1815.

49. Idem, p. xi.

50. Éric Baratay, Bêtes de somme, des animaux au service de l'homme, Paris, seuil, 2011 et Daniel Roche le montre à propos des chevaux dans les guerres nationales : La gloire et la puissance, Histoire de la culture équestre $X V I^{e}-X I X^{e}$ siècle, Paris, Paris, Fayard, 2011, p. 316-320. 
déborde les opérations physiques des organes pour s'étendre au "système intelligent et moral propre à chaque nature sensible ${ }^{51}$. Considérés depuis les impératifs économiques comme thérapeutiques, les animaux deviennent "nos utiles compagnons", des membres de la "famille humaine», des amis et des frères. Leur condition doit alors s'en trouver changée. "Ce n'est point assez d'éviter à l'égard de nos compagnons et de nos aides, tout mauvais traitement sans objet : soyons plus justes, cherchons à les rendre heureux " ${ }^{52}$. Du cercle d'Auteuil, le projet descend profondément dans les milieux vétérinaires. Lorsqu'en 1804, les directeurs et professeurs de l'École vétérinaire d'Alfort voulant profiter d'un changement à la tête du ministère de l'Intérieur adressent un mémoire au gouvernement et à l'opinion pour promouvoir les vétérinaires, ils se font vecteur et écho d'une troisième pensée liant un projet zootechnique à l'amélioration du sort des animaux. Le titre de leur ouvrage (Des moyens de rendre l'art vétérinaire plus utile), la citation en exergue ("la conservation des animaux utiles et le perfectionnement de leurs races seront toujours de la plus haute importance aux yeux d'une sage administration") et les premiers développements renvoient en effet clairement à une pensée associant besoin humain et condition de l'animal qui aboutit à une relecture de l'histoire vétérinaire liant dans une même dynamique, l'agronomie et la promotion de nos «utiles compagnons». "Soit par attachement, soit par spéculation, l'homme devoit tous ses soins à ces êtres intéressans qu'il a soumis à son empire, qu'il associe à ses travaux, dont les services et les produits divers contribuent aux jouissances de la société „"53. Un vétérinaire lyonnais François Aygalenq adapte jusqu’à la paraphrase la pensée de Cabanis. Il en nourrit son projet de réforme des études vétérinaires. Mais ni le projet des professeurs d'Alfort, ni celui de leur confrère lyonnais ne sont entendus. La relecture du concours de l'Institut de 1802 le confirme éloquemment ${ }^{54}$.

\section{UNE PENSÉE QUi SE RETOURNE CONTRE ELLE-MÊME}

Le XIX siècle, loin d'accomplir l'utopie des ménageries ou de l'économie rurale, voit apparaître des blocages techniques et des ruptures sociales et politiques. Le monde animal, par son activité économique, par sa présence nouvelle, par son étude constituée en champ singulier, par sa médecine spécifique, n’a pas seulement proposé la possibilité de «réparer les fléaux de la nature et les crimes de la politique», pour reprendre la formule du conven-

51. Pierre-Jean-Georges Cabanis, Rapports du physique et $d u$ moral, huitième mémoire : de l'influence du régime sur les habitudes morales. Troisième édition, tome second, Paris, chez Caille et Ravier, 1815 , p. 7.

52. Pierre-Jean-Georges Cabanis, Coup d'ail sur les révolutions et sur la réforme de la médecine, Paris, imprimerie de Crapelet, an XII-1804, p. 402.

53. P. Chabert et C.M.F. Fromage, Des moyens de rendre l'art vétérinaire plus utile, Paris, Marchant, brumaire an XIII p. i.

54. Pierre Serna, l'animal en République, op. cit. 
tionnel Joseph Eschasseriaux ${ }^{55}$. Il a aussi fourni un système de classification en remplacement de la société d'ordre abolie où la dimension biologique et les assignations surdéterminées par les arbres généalogiques renforcent les inégalités ${ }^{56}$. En clair, l'étude du monde animal a aidé à construire un ordre républicain. Au sortir de la Terreur et de son traumatisme, les naturalistes produisent des modalités d'action et des clés de lectures rationnelles pour comprendre un monde qui est devenu soudainement d'une complexité extrême avec l'effondrement des repères de l'Ancien Régime et l'apparition d'une société difficile à décrypter ${ }^{57}$. Le monde des animaux offre des catégorisations politiques d'une rassurante efficacité, entre les bêtes féroces assoiffées de sang et les travailleurs modestes et courageux, entre le monde des animaux utiles et des nuisibles, des excentriques et des normaux, des féconds et des stériles, des rois d'avant et des modestes d'aujourd'hui, entre les domestiques et les sauvages, les barbares et les pacifiques. La floraison des pamphlets antijacobins qui font de Paris une immense ménagerie remplie d'hommes animaux, bien connus grâce au vocabulaire des savants divulgué et vulgarisé dans le public, après 1795 , contribue fortement à la disqualification des ennemis. Encore davantage, elle construit littéralement des modes de classements des différents groupes sociaux. À côté des êtres humains, citoyens qui payent le cens, propriétaires en voie de notabilité, futurs soutiens du premier consul, une masse grouille, une fourmilière ou une meute, c'est selon, s'agite. Il s'agit de contenir ce troupeau qui peut se muer en bêtes féroces. Avant d'être dangereuses et laborieuses, ces classes-là sont à peine sorties d'une animalité inquiétante menée par des hommes-tigres qu'il s'agit d'encager sans pitiés8. Les penseurs de la Contre-révolution ne s'y sont pas trompés qui s'ingénient à décrire une régression animale dans le statut des nouveaux citoyens, lorsque l'abbé Barruel évoque en un néologisme parlant, la «férocisation» des mours républicaines, marginalisant les citoyens les plus démunis, les abêtissant au sens littéral du terme, stigmatisant les militants démocrates, les bestialisant pour longtemps ${ }^{59}$. Désormais, les espaces sont renversés : les bêtes sont dans la ville et Paris est devenue un zoo dangereux pour les «honnêtes gens», alors que la ménagerie s'efforce de ressembler à une ferme républicaine pour un public à éduquer, à dresser.

55. Eschasseriaux, Rapport au nom du comité d'agriculture, Imprimé par ordre de la Convention nationale, de l'imprimerie nationale.

56. Dominique Guillo, Les figures de l'organisation. Sciences de la vie et sciences sociales au XIX'e siècle, Paris, PUF, 2003.

57. Alain Corbin, "Le XIX siècle ou la nécessité de l'assemblage», in Alain Corbin et alii [dir.], L'invention du XIX siècle : le XIXe siècle vu par lui-même (littérature, histoire, société), Paris, Klincksieck / Presses de la Sorbonne Nouvelle, 1999, p. 153-159.

58. Par exemple, Pétition du diable aux deux conseils demandant l'établissement d'une ménagerie dans la cour du palais du Directoire exécutif, et dans les jardins du conseil des Anciens et des Cinq-Cents. s. d. de l'imprimerie de la rue des Orties p. 478.

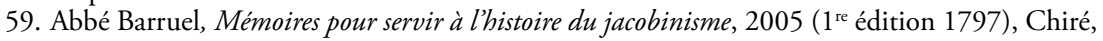


L'angle mort révolutionnaire et républicain relève de diverses constructions qui finissent par faire système : des attaques contre la «machine à terreur", contre son "paravent" régénérateur $\mathrm{d}^{\prime} \mathrm{abord}^{60}$, des attaques contre la nouvelle société née de la Révolution mais aussi de la capacité de l'élevage contemporain à dire ce qu'est l'élevage et son passéb1. Une histoire critique mais non érudite ressort enfin de choix historiographiques et d'une sousestimation de la littérature agronomique. C'est pourtant dans cette période révolutionnaire et dans l'ensemble de cette littérature tournée non vers les animaux mais vers le propriétaire et la société, pour créer les conditions de l'égalité des droits de l'homme, par une écologie républicaine, qu'on peut trouver une prise en compte originale et presque émancipatrice de la bête exploitée. À condition de penser l'égalité de l'homme avec ses semblables. Le discours des sciences de la nature, en élevant les animaux à la ressemblance des hommes, ne fait-il pas peser sur les plus fragiles ou les plus stigmatisés de ces derniers le risque d'un déclassement dans une proximité animale? Une chose est la pensée originale de l'animal au cœur des études en sciences sociales dans une perspective environnementale. Autre chose est une étude des animaux qui centrerait son attention sur les non-humains et s'éloignerait de la construction des systèmes de domination.

\section{Malik Mellah est doctorant en histoire à l'université Paris 1 Panthéon-Sorbonne; Pierre Serna est professeur d'histoire de la Révolution française à l'Université Paris 1 Panthéon-Sorbonne}

60. Cf. la mise au point dans Jean-Luc Chappey, Bernard Gainot, Guillaume Mazeau, Frédéric Régent, Pierre Serna, Pour quoi faire la Révolution, Marseille, Agone, 2012.

61. «Le credo scientifique et technique que défend Sanson s'inscrit dans le droit fil des Lumières. En pensant que le progrès de la science conduit nécessairement à un progrès de la vie morale, Sanson veut servir le progrès social en agriculture", Jocelyne Porcher, Bien-être animal et travail en élevage, Paris-Dijon, éducagri Inra, 2004, p. 43. 